\title{
Parallel Analysis in MDAnalysis using the Dask Parallel Computing Library
}

\author{
Mahzad Khoshlessan ${ }^{\ddagger}$, loannis Paraskevakos ${ }^{\S}$, Shantenu Jha ${ }^{\S}$, Oliver Beckstein ${ }^{\ddagger *}$
}

\begin{abstract}
The analysis of biomolecular computer simulations has become a challenge because the amount of output data is now routinely in the terabyte range. We evaluated if this challenge can be met by a parallel map-reduce approach with the Dask parallel computing library for task-graph based computing coupled with our MDAnalysis Python library for the analysis of molecular dynamics (MD) simulations. We performed a representative performance evaluation, taking into account the highly heterogeneous computing environment that researchers typically work in together with the diversity of existing file formats for MD trajectory data. We found that the underlying storage system (solid state drives, parallel file systems, or simple spinning platter disks) can be a deciding performance factor that leads to data ingestion becoming the primary bottleneck in the analysis work flow. However, the choice of the data file format can mitigate the effect of the storage system; in particular, the commonly used Gromacs XTC trajectory format, which is highly compressed, can exhibit strong scaling close to ideal due to trading a decrease in global storage access load against an increase in local per-core CPU-intensive decompression. Scaling was tested on a single node and multiple nodes on national and local supercomputing resources as well as typical workstations. Although very good strong scaling could be achieved for single nodes, good scaling across multiple nodes was hindered by the persistent occurrence of "stragglers", tasks that take much longer than all other tasks, and whose ultimate cause could not be completely ascertained. In summary, we show that, due to the focus on high interoperability in the scientific Python eco system, it is straightforward to implement map-reduce with Dask in MDAnalysis and provide an in-depth analysis of the considerations to obtain good parallel performance on HPC resources.
\end{abstract}

Index Terms-MDAnalysis, High Performance Computing, Dask, Map-Reduce, MPI for Python

\section{Introduction}

MDAnalysis is a Python library that provides users with access to raw simulation data and enables structural and temporal analysis of molecular dynamics (MD) trajectories generated by all major MD simulation packages [GLB $\left.{ }^{+} 16\right]$, [MADWB11]. MD trajectories are time series of positions (and sometimes also velocities) of the simulated atoms or particles; using statistical mechanics one can calculate experimental observables from these time series [FS02], [MM14]. The size of these trajectories is growing as the simulation times are being extended beyond micro-seconds and larger systems with increasing numbers of atoms are simulated. The amount of data to be analyzed is growing rapidly into the

* Arizona State University, Department of Physics, Tempe, AZ 85287, USA § RADICAL, ECE, Rutgers University, Piscataway, NJ 08854, USA *Corresponding author: obeckste@asu.edu

Copyright $\odot 2017$ Mahzad Khoshlessan et al. This is an open-access article distributed under the terms of the Creative Commons Attribution License, which permits unrestricted use, distribution, and reproduction in any medium, provided the original author and source are credited. terabyte range and analysis is increasingly becoming a bottleneck in MD workflows [CR15]. Therefore, there is a need for high performance computing (HPC) approaches for the analysis of MD trajectory data $\left[\mathrm{TRB}^{+} 08\right.$ ], [RCI13].

MDAnalysis does not yet provide a standard interface for parallel analysis; instead, various existing parallel libraries such as Python multiprocessing, joblib, and mpi4py [DPS05], [DPKC11] are currently used to parallelize MDAnalysis-based code on a case-by-case basis. Here we evaluated performance for parallel map-reduce [DG08] type analysis with the Dask parallel computing library [Roc15] for task-graph based distributed computing on HPC and local computing resources. Although Dask is able to implement much more complex computations than map-reduce, we chose Dask for this task because of its ease of use and because we envisage using this approach for more complicated analysis applications whose parallelization cannot be easily expressed as a simple map-reduce algorithm.

As the computational task we performed a common task in the analysis of the structural dynamics of proteins: we computed the time series of the root mean squared distance (RMSD) of the positions of all $\mathrm{C}_{\alpha}$ atoms to their initial coordinates at time 0 ; for each time step ("frame") in the trajectory, rigid body degrees of freedom (translations and rotations) have to be removed through an optimal structural superposition that minimizes the RMSD [MM14] (Figure 1). A range of commonly used MD file formats (CHARMM/NAMD DCD [BBIM ${ }^{+}$09], Gromacs XTC $\left[\mathrm{AMS}^{+} 15\right]$, Amber NCDF $\left.\left[\mathrm{CCD}^{+} 05\right]\right)$ and different trajectory sizes were benchmarked.

We looked at different HPC resources including national

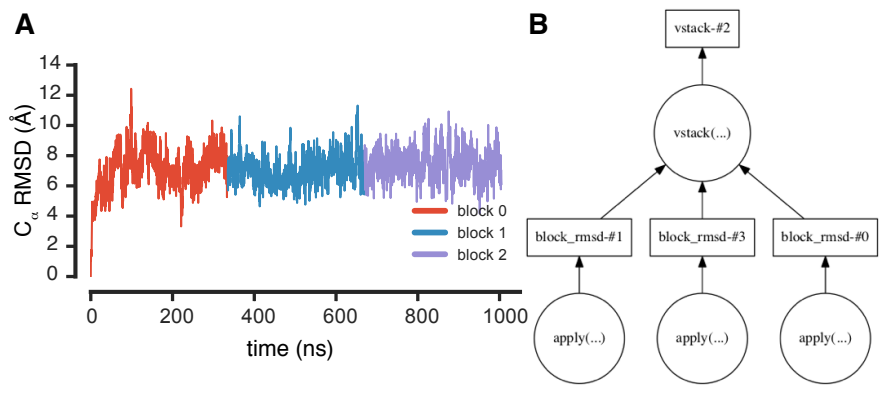

Fig. 1: Calculation of the root mean square distance (RMSD) of a protein structure from the starting conformation via map-reduce with Dask. A RMSD as a function of time, with partial time series colored by trajectory block. B Dask task graph for splitting the RMSD calculation into three trajectory blocks. 
supercomputers (XSEDE TACC Stampede and SDSC Comet), university supercomputers (Arizona State University Research Computing Saguaro), and local resources (Gigabit networked multi-core workstations). The tested resources are parallel and heterogeneous with different CPUs, file systems, high speed networks and are suitable for high-performance distributed computing at various levels of parallelization. Different storage systems such as solid state drives (SSDs), hard disk drives (HDDs), network file system (NFS), and the parallel Lustre file system (using HDDs) were tested to examine the effect of $\mathrm{I} / \mathrm{O}$ on the performance. The benchmarks were performed both on a single node and across multiple nodes using the multiprocessing and distributed schedulers in the Dask library.

We previously showed that the overall computational cost scales directly with the length of the trajectory, i.e., the weak scaling is close to ideal and is fairly independent from other factors [KB17]. Here we focus on the strong scaling behavior, i.e., the dependence of overall run time on the number of CPU cores used. Competition for access to the same file from multiple processes appears to be a bottleneck and therefore the storage system is an important determinant of performance. But because the trajectory file format dictates the data access pattern, overall performance also depends on the actual data format, with some formats being more robust against storage system specifics than others. Overall, good strong scaling performance could be obtained for a single node but robust across-node performance remained challenging. In order to identify performance bottlenecks we examined several other factors including the effect of striping in the parallel Lustre file system, over-subscribing (using more tasks than Dask workers), the performance of the Dask scheduler itself, and we also benchmarked an MPI-based implementation in contrast to the Dask approach. From these tests we tentatively conclude that poor across-nodes performance is rooted in contention on the shared network that may slow down individual tasks and lead to poor load balancing. Nevertheless, Dask with MDAnalysis appears to be a promising approach for high-level parallelization for analysis of MD trajectories, especially at moderate CPU core numbers.

\section{Methods}

We implemented a simple map-reduce scheme to parallelize processing of trajectories over contiguous blocks. We tested libraries in the following versions: MDAnalysis 0.15.0, Dask 0.12.0 (also 0.13.0), distributed 1.14.3 (also 1.15.1), and NumPy 1.11.2 (also 1.12.0) [VCV11].

import numpy as np

import MDAnalysis as mda

from MDAnalysis.analysis.rms import rmsd

The trajectory is split into n_blocks blocks with inital frame start and final frame stop set for each block. The calculation on each block (function block_rmsd (), corresponding to the map step) is delayed with the delayed ( ) function in Dask:

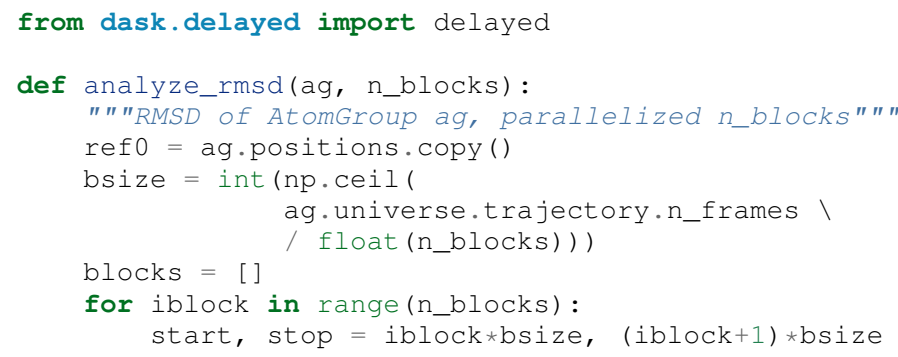

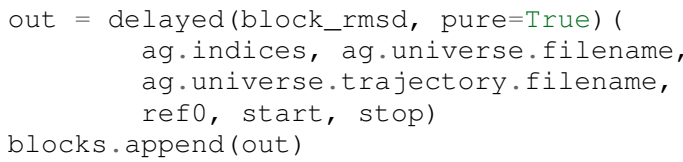

In the reduce step, the partial time series from each block are concatenated in the correct order (np.vstack, see Figure 1 A); because results from delayed objects are used, this step also has to be delayed.

As computational load we implement the calculation of the root mean square distance (RMSD) of the $\mathrm{C}_{\alpha}$ atoms of the protein adenylate kinase [SB14] when fitted to a reference structure using an optimal rigid body superposition [MM14], using the qcprot implementation [LAT10] in MDAnalysis [GLB $\left.{ }^{+} 16\right]$. The RMSD is calculated for each trajectory frame in each block by iterating over u.trajectory [start:stop]:

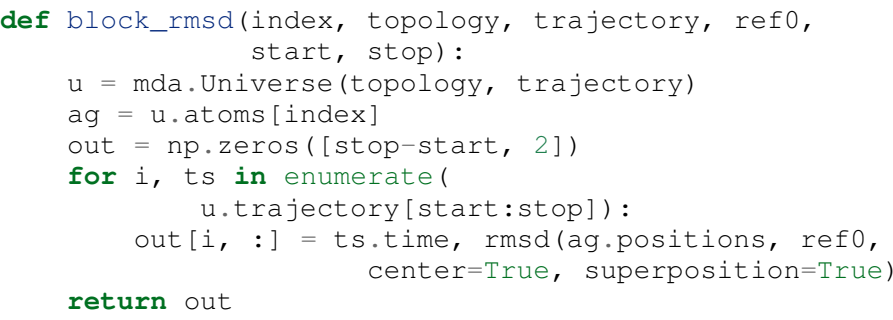

Dask produces a task graph (Figure $1 \mathrm{~B}$ ) and the computation of the graph is executed in parallel through a Dask scheduler such as dask.multiprocessing (or dask.distributed):

from dask.multiprocessing import get

$\mathrm{u}=$ mda. Universe (PSF, DCD)

ag $=u . s e l e c t \_a t o m s(" p r o t e i n$ and name CA")

result $=$ analyze_rmsd(ag, n_blocks)

timeseries $=$ result.compute (get=get)

The complete code for benchmarking as well as an alternative implementation based on mpi4py is available from https://github.com/Becksteinlab/Parallel-analysis-in-theMDAnalysis-Library under the MIT License.

The data files consist of a topology file adk4AKE.psf (in CHARMM PSF format; $N=3341$ atoms) and a trajectory lake_007-nowater-core-dt240ps.dcd (DCD format) of length $1.004 \mu$ s with 4187 frames; both are freely available from figshare at DOI 10.6084/m9.figshare.5108170 [SB17]. Files in XTC and NCDF formats are generated from the DCD on the fly using MDAnalysis. To avoid operating system caching, files were copied and only used once for each benchmark. All results for Dask distributed were obtained across three nodes on different clusters.

Trajectories with different number of frames per trajectory were analyzed to assess the effect of trajectory file size. These trajectories were generated by concatenating the base trajectory 50 , 100, 300, and 600 times and are referred to as, e.g., "DCD300x" or "XTC600x". Run time was analyzed on single nodes (1-24 CPU cores) and up to three nodes (1-72 cores) as function of the number of cores (strong scaling behavior) and trajectory sizes (weak scaling). However, here we only present strong scaling data for the 300x and 600x trajectory sizes, which represent typical medium size results. For an analysis of the full data including weak scaling results set see the Technical Report [KB17].

The DCD file format is a binary representation for 32-bit floating point numbers (accuracy of positions about $10^{-6} \AA$ ) and 
the DCD300x trajectory has a file size of 47 GB (DCD600x is twice as much); XTC is a lossy compressed format that effectively rounds floats to the second decimal (accuracy about $10^{-2} \AA$, which is sufficient for typical analysis) and XTC300x is only 15 GB. Amber NCDF is implemented with netCDF classic format version 3.6.0 (same accuracy as DCD) and trajectories are about the same size as DCD. DCD and NCDF natively allow fast random access to frames or blocks of frames, which is critical to implement the map-reduce algorithm. XTC does not natively support frame seeking but MDAnalysis implements a fast frame scanning algorithm for XTC files that caches all frame offsets and so enables random access for the XTC format, too $\left[\mathrm{GLB}^{+} 16\right]$. In MDAnalysis 0.15.0, Amber NCDF files are read with the Python netCDF4 module that wraps the netcdf $\mathrm{C}$ library; in the upcoming MDAnalysis 0.17.0, netCDF v3 files are read with the pure Python scipy.io.netcdf module, which tends to read netCDF v3 files about five times faster than netCDF4, and hence results for NCDF presented here might change with more recent versions of MDAnalysis.

Performance was quantified by measuring the average time per trajectory frame to load data from storage into memory (I/O time per frame, $t_{\mathrm{I} / \mathrm{O}}$ ), the average time to complete the RMSD calculation (compute time per frame, $t_{\text {comp }}$ ), and the total wall time for job execution $t_{N}$ when using $N$ CPU cores. Strong scaling was assessed by calculating the speed up $S(N)=t_{1} / t_{N}$ and the efficiency $E(N)=S(N) / N$.

\section{Results and Discussion}

Trajectories from MD simulations record snapshots of the positions of all particles at regular time intervals. A snapshot at a specified time point is called a frame. MDAnalysis only loads a single frame into memory at any time [GLB $\left.{ }^{+} 16\right]$, [MADWB11] to allow the analysis of large trajectories that may contain, for example, $n_{\text {frames }}=10^{7}$ frames in total. In a map-reduce approach, $N$ processes will iterate in parallel over $N$ chunks of the trajectory, each containing $n_{\text {frames }} / N$ frames. Because frames are loaded serially, the run time scales directly with $n_{\text {frames }}$ and the weak scaling behavior (as a function of trajectory length) is trivially close to ideal as seen from the data in [KB17]. Weak scaling with the system size also appears to be fairly linear, according to preliminary data (not shown). Therefore, in the following we focus exclusively on the harder problem of strong scaling, i.e., reducing the run time by employing parallelism.

\section{Effect of File Format on I/O Performance}

We first sought to quantify the effect of the trajectory format on the analysis performance. The overall run time depends strongly on the trajectory file format as well as the underlying storage system as shown for the 300x trajectories in Figure 2; results for other trajectory sizes are similar (see [KB17]) except for the smallest $50 \mathrm{x}$ trajectories where possibly caching effects tend to improve overall performance. Using DCD files with SSDs on a single node (Figure 2 A) is about one order of magnitude faster than the other formats (Figure $2 \mathrm{~B}, \mathrm{C}$ ) and scales near linearly for small CPU core counts $(N \leq 12)$. However, DCD does not scale at all with other storage systems such as HDD or NFS and run time only improves up to $N=4$ on the Lustre file system. On the other hand, the run time with NCDF and especially with XTC trajectories improves linearly with increasing $N$, with XTC on Lustre and $N=24$ cores almost obtaining the best DCD run time of about 30
A

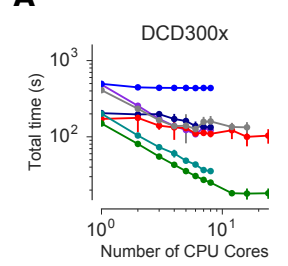

B

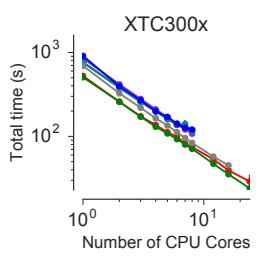

D

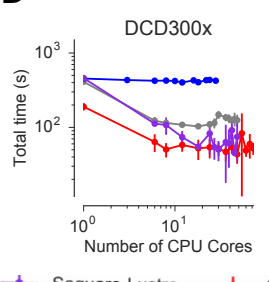

E

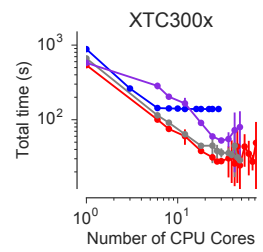

$\mathbf{F}$
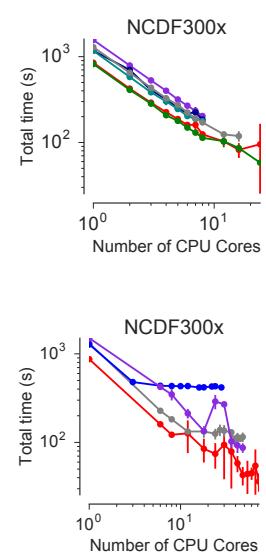

+ Local-HDD

$\rightarrow$ Comet-Lustre

$\rightarrow$ Comet-SSD

Number of CPU Cores

$\multimap$ Local-Remote-HDD

Fig. 2: Comparison of total job execution time $t_{N}$ for different file formats (300x trajectory size) using Dask multiprocessing on a single node (1-24 CPU cores, $\boldsymbol{A}-\boldsymbol{C}$ ) and Dask distributed on up to three nodes (1-72 CPU cores, $\boldsymbol{D}-\boldsymbol{F})$. The trajectory was split into $M$ blocks and computations were performed using $N=M C P U$ cores. The runs were performed on different resources (ASU RC Saguaro, SDSC Comet, TACC Stampede, local workstations with different storage systems (locally attached HDD, remote HDD (via network file system, NFS), locally attached SSD, Lustre parallel file system with a single stripe). A, D CHARMM/NAMD DCD. B, E Gromacs $X T C . \boldsymbol{C}, \boldsymbol{F}$ Amber NetCDF.
A

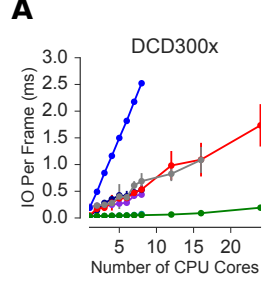

D

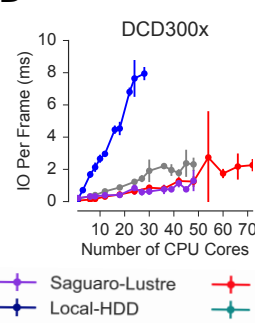

B

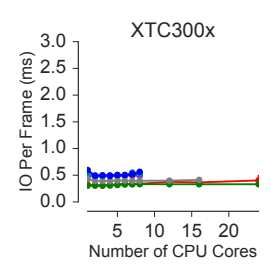

$\mathbf{E}$

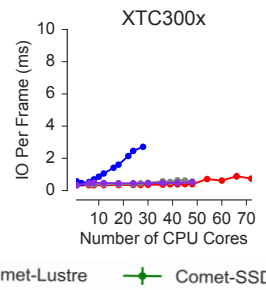

C

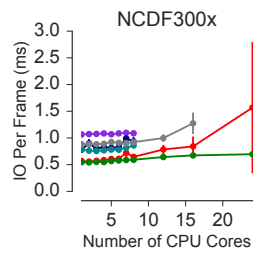

$\mathbf{F}$

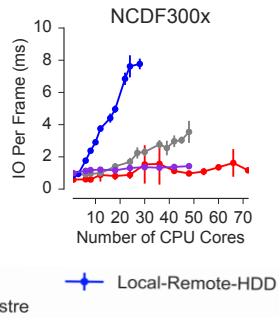

Fig. 3: Comparison of I/O time $t_{I / O}$ per frame between different file formats (300x trajectory size) using Dask multiprocessing on a single node $(\boldsymbol{A}-\boldsymbol{C})$ and Dask distributed on multiple nodes $(\boldsymbol{D}-\boldsymbol{F}) . \boldsymbol{A}, \boldsymbol{D}$ CHARMM/NAMD DCD. B, E Gromacs XTC. C, F Amber NetCDF. All parameters as in Fig. 2.

s (SSD, $N=12)$; at the highest single node core count $N=24$, XTC on SSD performs even better (run time about $25 \mathrm{~s}$ ). For larger $N$ on multiple nodes, only a shared file system (Lustre or NFS) based on HDD was available. All three file formats only show small improvements in run time at higher core counts $(N>24)$ on the Lustre file system on supercomputers with fast interconnects and no improvements on NFS over Gigabit (Figure 2 D-F).

In order to explain the differences in performance and scaling of the file formats, we analyzed the time to load the coordinates 


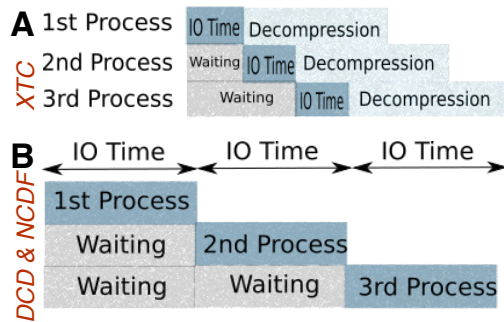

Fig. 4: I/O pattern for reading frames in parallel from commonly used MD trajectory formats. A Gromacs XTC file format. B CHARMM/NAMD DCD file format and Amber NCDF format.

of a single frame from storage into memory $\left(t_{\mathrm{I} / \mathrm{O}}\right)$ and the time to perform the computation on a single frame using the in-memory data $\left(t_{\text {comp }}\right)$. As expected, $t_{\text {comp }}$ is independent from the file format, $n_{\text {frames }}$, and $N$ and only depends on the CPU type itself (mean and standard deviation on SDSC Comet $0.098 \pm 0.004 \mathrm{~ms}$, TACC Stampede $0.133 \pm 0.000 \mathrm{~ms}$, ASU RC Saguaro $0.174 \pm 0.000$ $\mathrm{ms}$, local workstations $0.225 \pm 0.022 \mathrm{~ms}$, see [KB17]). Figure 3, however shows how $t_{\mathrm{I} / \mathrm{O}}$ (for the $300 \mathrm{x}$ trajectories) varies widely and in most cases, is at least an order of magnitude larger than $t_{\text {comp }}$. The exception is $t_{\mathrm{I} / \mathrm{O}}$ for the DCD file format using SSDs, which remains small $(0.06 \pm 0.04 \mathrm{~ms}$ on SDSC Comet $)$ and almost constant with $N \leq 12$ (Figure $3 \mathrm{~A}$ ) and as a result, the DCD file format shows good scaling and the best performance on a single node. For HDD-based storage, the time to read data from a DCD frame increases with the number of processes that are simultaneously trying to access the DCD file. XTC and NCDF show flat $t_{\mathrm{I} / \mathrm{O}}$ with $N$ on a single node (Figure $3 \mathrm{~B}, \mathrm{C}$ ) and even for multiple nodes, the time to ingest a frame of a XTC trajectory is almost constant, except for NFS, which broadly shows poor performance (Figure $3 \mathrm{E}, \mathrm{F}$ ).

Depending on the file format the loading time of frames into memory will be different, as illustrated in Figure 4. The XTC file format is compressed and has a smaller file size when compared to the other formats. When a compressed XTC frame is loaded into memory, it is immediately decompressed (see Figure 4 A). During decompression by one process, the file system allows the next process to load its requested frame into memory. As a result, competition for file access between processes and overall wait time is reduced and $t_{\mathrm{I} / \mathrm{O}}$ remains almost constant, even for large number of parallel processes (Figure $3 \mathrm{~B}, \mathrm{E}$ ). Neither DCD nor NCDF files are compressed and multiple processes compete for access to the file (Figure $4 \mathrm{~B}$ ) although NCDF files is a more complicated file format than DCD and has additional computational overhead. Therefore, for DCD the I/O time per frame is very small as compared to other formats when the number of processes is small (and the storage is fast), but even at low levels of parallelization, $t_{\mathrm{I} / \mathrm{O}}$ increases due to the overlapping of per frame trajectory data access (Figure 3 A, D). Data access with NCDF is slower but due to the additional computational overhead, is amenable to some level of parallelization (Figure $3 \mathrm{C}, \mathrm{F}$ ).

\section{Strong Scaling Analysis for Different File Formats}

We quantified the strong scaling behavior by analyzing the speedup $S(N)$; as an example, the 300x trajectories for multiprocessing and distributed schedulers are show in Figure 5. The DCD format exhibits poor scaling, except for $N \leq 12$ on a single node and SSDs (Figure $5 \mathrm{~A}, \mathrm{D}$ ) and is due to the increase in $t_{\mathrm{I} / \mathrm{O}}$ with $N$, as
A

B

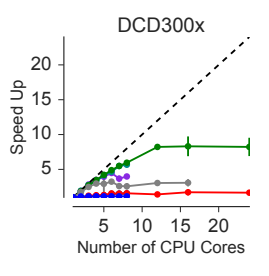

D
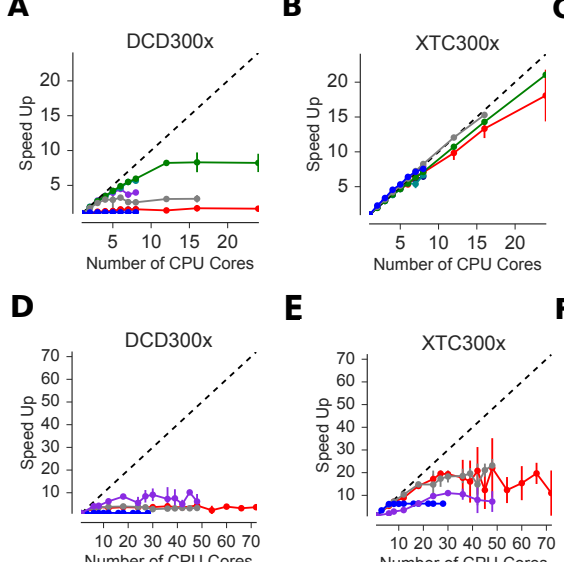

C

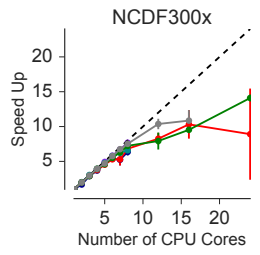

E
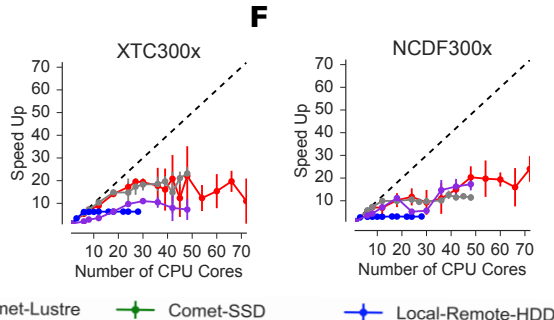

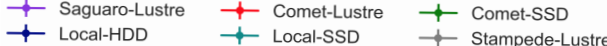

Fig. 5: Speed-up $S$ for the analysis of the 300x trajectory on HPC resources using Dask multiprocessing (single node, $\boldsymbol{A}-\boldsymbol{C}$ ) and distributed (up to three nodes, $\boldsymbol{D}-\boldsymbol{F}$ ). The dashed line shows the ideal limit of strong scaling. All other parameters as in Fig. 2.
A

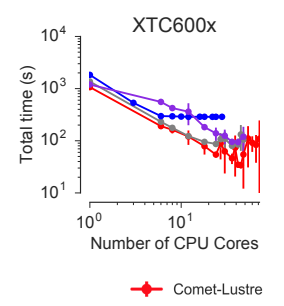

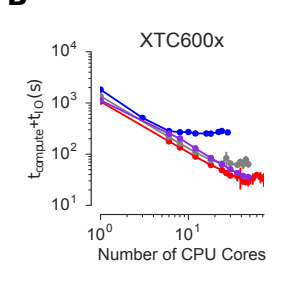

C

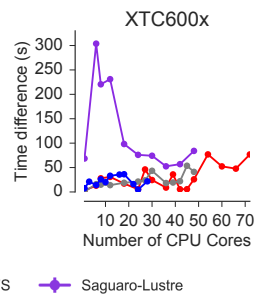

Fig. 6: Detailed analysis of timings for the 600x XTC trajectory on $H P C$ resources using Dask distributed. All other parameters as in Fig. 2. A Total time to solution (wall clock), $t_{N}$ for $N$ trajectory blocks using $N_{\text {cores }}=N C P U$ cores. B Sum of the I/O time per frame $t_{I / O}$ and the (constant) time for the RMSD computation $t_{\text {comp }}$ (data not shown). C Difference $t_{N}-n_{\text {frames }}\left(t_{I / O}+t_{\text {comp }}\right)$, accounting for the cost of communications and other overheads.

discussed in the previous section. The XTC file format scales close to ideal on $N \leq 24$ cores (single node) for both the multiprocessing and distributed scheduler, almost independent from the underlying storage system. The NCDF file format only scales well up to 8 cores (Figure $5 \mathrm{C}, \mathrm{F}$ ) as expected from $t_{\mathrm{I} / \mathrm{O}}$ in Figure $3 \mathrm{C}, \mathrm{F}$.

For the XTC file format, $t_{\mathrm{I} / \mathrm{O}}$ is is nearly constant up to $N=50$ cores (Figure $3 \mathrm{E}$ ) and $t_{\text {comp }}$ also remains constant up to 72 cores. Therefore, close to ideal scaling would be expected for up to 50 cores, assuming that average processing time per frame $t_{\text {comp }}+t_{\mathrm{I} / \mathrm{O}}$ dominates the computation. However, based on Figure $5 \mathrm{E}$, the XTC format only scales well up to about 24 cores, which suggests that this assumption is wrong and there are other computational overheads.

To identify and quantify these additional overheads, we analyzed the performance of the XTC600x trajectory in more detail (Figure 6); results for other trajectory sizes are qualitatively similar. The total job execution time $t_{N}$ differs from the total compute and I/O time, $N\left(t_{\text {comp }}+t_{\mathrm{I} / \mathrm{O}}\right)$. This difference measures additional overheads that we did not consider so far. It increases with trajectory size for all file formats and for all machines (for details refer to [KB17]) but is smaller for SDSC Comet and TACC 


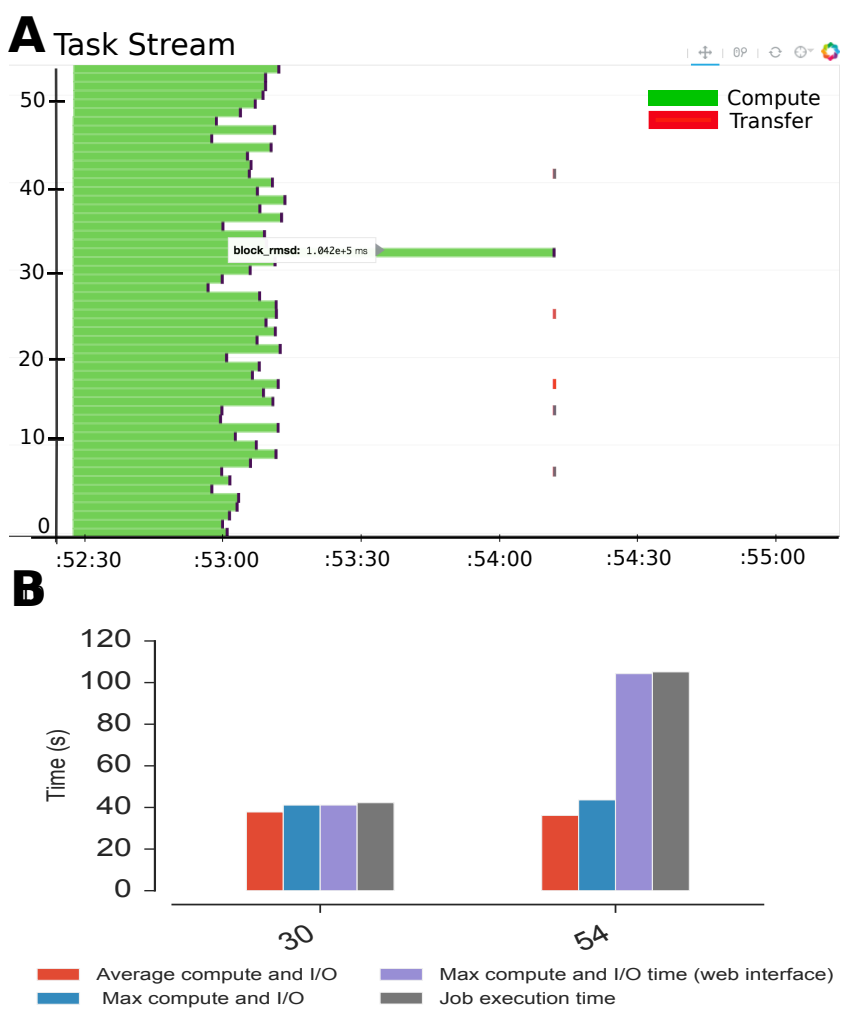

Fig. 7: Evidence for uneven distribution of task execution times, shown for the XTC600x trajectory on SDSC Comet on the Lustre file system. A Task stream plot showing the fraction of time spent on different parts of the task by each worker, obtained using the Dask web-interface. (54 tasks for 54 workers that used $N=54$ cores). Green bars ("Compute") represent time spent on RMSD calculations, including trajectory $I / O$, red bars show data transfer. A "straggler" task (\#32) takes much longer than any other task and thus determines the total execution time. $\boldsymbol{B}$ Comparison between timing measurements from instrumentation inside the Python code (average compute and I/O time per task $n_{\text {frames }} / N\left(t_{\text {comp }}+t_{I / O}\right), \max \left[n_{\text {frames }} / N\left(t_{\text {comp }}+t_{I / O}\right)\right]$, and $\left.t_{N}\right)$ and Dask web-interface for $N=30$ and $N=54$ cores.

Stampede than compared to other machines. The difference is small for the results obtained using the multiprocessing scheduler on a single node but it is substantial for the results obtained using distributed scheduler on multiple nodes.

In order to obtain more insight into the underlying network behavior both at the Dask worker level and communication level and in order to pinpoint the origin of the overheads, we used the web-interface of the Dask library, which is launched together with the Dask scheduler. Dask task stream plots such as the example shown in Figure 7 A typically show one or more straggler tasks that take much more time than the other tasks and as a result slow down the whole run. Stragglers do not actually spend more time on the RMSD computation and trajectory I/O than other tasks, as shown by comparing the average compute and I/O time for a single task $i, n_{\text {frames }} / N\left(t_{\mathrm{comp}, i}+t_{\mathrm{I} / \mathrm{O}, i}\right)$, with the maximum over all tasks $\max _{i}\left[n_{\text {frames }} / N\left(t_{\mathrm{comp}, i}+t_{\mathrm{I} / \mathrm{O}, i}\right)\right]$ (Figure $7 \mathrm{~B}$ ). These stragglers are observed at some repeats when the number of cores is more than 24 . However, we do not always see these stragglers which shows the importance of collecting statistics and looking at the average value of several repeats ( 5 in the present study). For example, for $N=30$ at one repeat no straggler was observed but, the statistics show poor perforrmance as also seen in Figure
$6 \mathrm{~A}$ and B. However, as seen in the example for $N=54$ for one repeat, the maximum compute and I/O time as measured inside the Python code is smaller than the maximum value extracted from the web-interface (and the Dask scheduler) (Figure 7 B). The maximum compute and I/O value from the scheduler matches the total measured run time, indicating that stragglers limit the overall performance of the run. The timing of the scheduler includes waiting due to network effects, which would explain why the difference is only visible when using multiple nodes where the node interconnect must be used.

\section{Challenges for Good HPC Performance}

All results were obtained during normal, multi-user, production periods on all machines, which means that jobs run times are affected by other jobs on the system. This is true even when the job is the only one using a particular node, which was the case in the present study. There are shared resources such as network file systems that all the nodes use. The high speed interconnect that enables parallel jobs to run is also a shared resource. The more jobs are running on the cluster, the more contention there is for these resources. As a result, the same job run at different times may take a different amount of time to complete, as seen in the fluctuations in task completion time across different processes. These fluctuations differ in each repeat and are dependent on the hardware and network. There is also variability in network latency, in addition to the variability in underlying hardware in each machine, which may also cause the results to vary across different machines. Since our map-reduce problem is pleasantly parallel, each or a subset of computations can be executed by independent processes. Furthermore, all of our processes have the same amount of work to do, namely one trajectory block per process, and therefore our problem should exhibit good load balancing. Therefore, observing the stragglers shown in Figure 7 $\mathrm{A}$ is unexpected and the following sections aim to identify possible causes for their occurrence.

\section{Performance Optimization}

We tested different features of the computing environment to identify causes of stragglers and to improve performance and robustness, focusing on the XTC file format as the most promising candidate so far. We tested the hypothesis that waiting for file access might lead to stalled tasks by increasing the effective number of accessible files through "striping" in the Lustre parallel file system. We investigated the hypothesis that the Dask distributed scheduler might be too slow to schedule the tasks and we looked at improved load balancing by over-subscribing Dask workers.

Effect of Lustre Striping: As discussed before, the overlapping of data requests from different processes can lead to higher $\mathrm{I} / \mathrm{O}$ time and as a result poor performance. $t_{\mathrm{I} / \mathrm{O}}$ strongly affects performance since it is much larger than $t_{\text {comp }}$ in all multi-node scenarios. Although the XTC format showed the best performance, for multiple nodes $t_{\mathrm{I} / \mathrm{O}}$ increased for it, too (Figure $3 \mathrm{E}$ ). In Lustre, a copy of the shared file can be in different physical storage devices (object storage targets, OSTs). Single shared files can have a stripe count equal to the number of nodes or processes which access the file. We set the stripe count equal to three, which is equal to the number of nodes used for our benchmark using the distributed scheduler. This might improve performance, since all the processes from each node will have a copy of the file and as a result the contention due to many data requests should decrease. Figure 8 show the speed up and I/O time per frame plots obtained 
$\mathbf{A}$

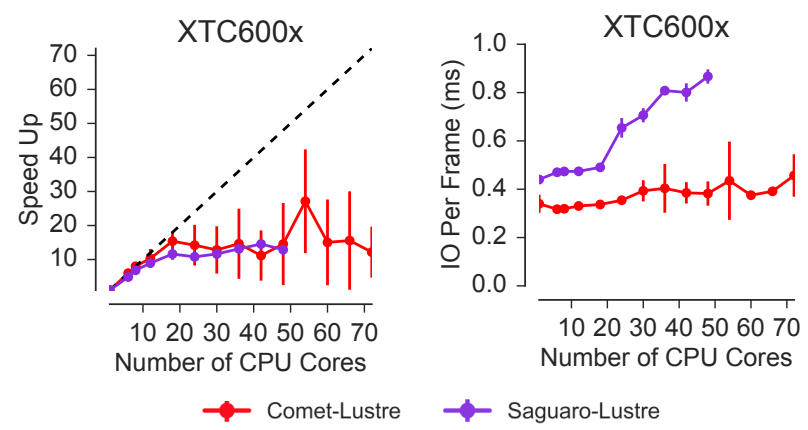

Fig. 8: Effect of striping with the Lustre distributed file system. The XTC600x trajectory was analyzed on HPC resources (ASU RC Saguaro, SDSC Comet) with Dask distributed and a Lustre stripe count of three, i.e., data were replicated across three servers. One trajectory block was assigned to each worker, i.e., the number of tasks equaled the number of CPU cores. A Speed-up. B Average I/O time per frame, $t_{I / O}$.
A

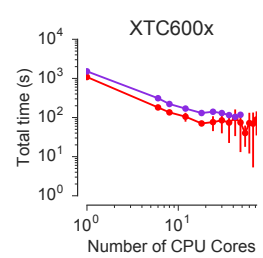

B

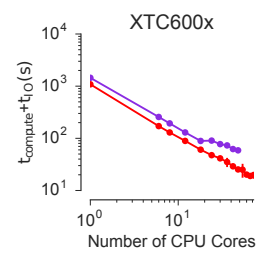

C

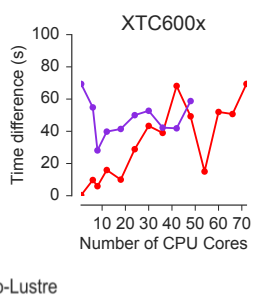

Fig. 9: Detailed timings for three-fold Lustre striping (see Fig. 8 for other parameters). A Total time to solution (wall clock), $t_{N}$ for $M$ trajectory blocks using $N=M C P U$ cores. $\boldsymbol{B} t_{\text {comp }}+t_{I / O}$, average sum of the I/O time ( $t_{I / O}$, Fig. $\left.8 \mathrm{~B}\right)$ and the (constant) time for the RMSD computation $t_{\text {comp }}$ (data not shown). C Difference $t_{N}-n_{\text {frames }}\left(t_{I / O}+\right.$ $\left.t_{\text {comp }}\right)$, accounting for communications and overheads that are not directly measured.

for XTC file format (XTC600x) when striping is activated. I/O time remains constant for up to 72 cores. Thus, striping improves $t_{\mathrm{I} / \mathrm{O}}$ and makes file access more robust. However, the timing plots in Figure 9 still show a time difference between average total compute and I/O time and job execution time that remains due to stragglers and as a result the overall speed-up is not improved.
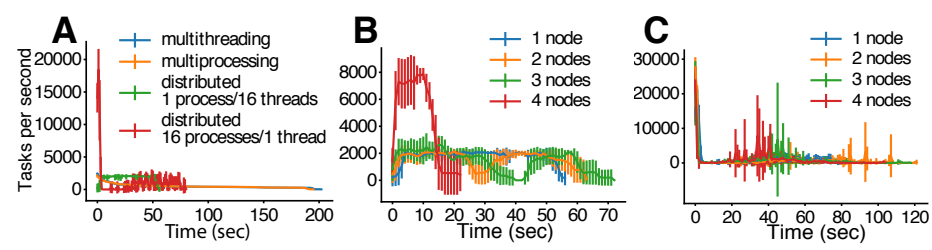

Fig. 10: Benchmark of Dask scheduler throughput on TACC Stampede. Performance is measured by the number of empty pass tasks that were executed in a second. The scheduler had to launch 100,000 tasks and the run ended when all tasks had been run. A single node with different schedulers; multithreading and multiprocessing are almost indistinguishable from each other. $\boldsymbol{B}$ multiple nodes with the distributed scheduler and 1 worker process per node. $C$ multiple nodes with the distributed scheduler and 16 worker processes per node.
Scheduler Throughput: In order to test the hypothesis that straggler tasks were due to limitations in the speed of the Dask scheduler, we performed scheduling experiments with all Dask schedulers (multithreaded, multiprocessing and distributed) on TACC Stampede (16 CPU cores per node). In each run, a total of 100,000 zero workload (pass) tasks were executed in order to measure the maximum scheduling throughput; each run itself was repeated ten times and mean values together with standard deviations were reported. Figure 10 A shows the throughput of each scheduler over time on a single Stampede node, with Dask scheduler and worker being located on the same node. The most efficient scheduler is the distributed scheduler, which manages to schedule 20,000 tasks per second when there is one worker process for each available core. The distributed scheduler with just one worker process and a number of threads equal to the number of available cores has lower peak performance of about 2000 tasks/s and is able to schedule and execute these 100,000 tasks in $50 \mathrm{~s}$. The multiprocessing and multithreading schedulers behave similarly, but need much more time (about $200 \mathrm{~s}$ ) to finish compared to distributed.

Figure $10 \mathrm{~B}$ shows the distributed scheduler's throughput over time for increasing number of nodes when each node has a single worker process and each worker launches a thread to execute a task (maximum 16 threads per worker). No clear pattern for the throughput emerges, with values between 2000 and 8000 tasks/s. Figure $10 \mathrm{C}$ shows the same execution with Dask distributed set up to have one worker process per core, i.e., 16 workers per node. The scheduler never reaches its steady throughput state, compared to Figure $10 \mathrm{~B}$ so that it is difficult to quantify the effect of the additional nodes. Although a peak throughput between 10,000 to 30,000 tasks/s is reported, overall scheduling is erratic and the total 100,000 tasks are not completed sooner than for the case with 1 worker per node with 16 threads. It appears that assigning one worker process to each core will speed up Dask's throughput but more work would need to be done to assess if the burst-like behavior seen in this case is an artifact of the zero workload test.

Either way, the distributed and even the multiprocessing scheduler are sufficiently fast as to not cause a bottleneck in our mapreduce problem and are probably not responsible for the stragglers.

Effect of Over-Subscribing: In order to make our code more robust against uncertainty in computation times we explored over-subscribing the workers, i.e., to submit many more tasks than the number of available workers (and CPU cores, using one worker per core). Over-Subscription might allow Dask to balance the load appropriately and as a result cover the extra time when there are some stragglers. We set the number $M$ of tasks to be three times the number of workers, $M=3 N$, where the number of workers $N$ equaled the number of CPU cores; now each task only works on $n_{\text {frames }} / M$ frames. To reduce the influence of $t_{\mathrm{I} / \mathrm{O}}$ on the benchmark, Lustre-striping was activated and set to three, equal to the number of nodes used.

For XTC600x, no substantial speed-up is observed due to oversubscribing (compare Figure 11 A to $8 \mathrm{~A}$ ), although fluctuations are reduced. As before, the I/O time is constant up to 72 cores due to striping (Figure $11 \mathrm{~B}$ ). However, a time difference between average total compute and I/O time and job execution time (Figure 12) reveals that over-subscribing does not help to remove the stragglers and as a result the overall speed-up is not improved. Figure 13 shows a time comparison for different parts of the calculations. The overhead in the calculations is small up to 24 cores (single node). For lower $N$, the largest fraction of time is 
A

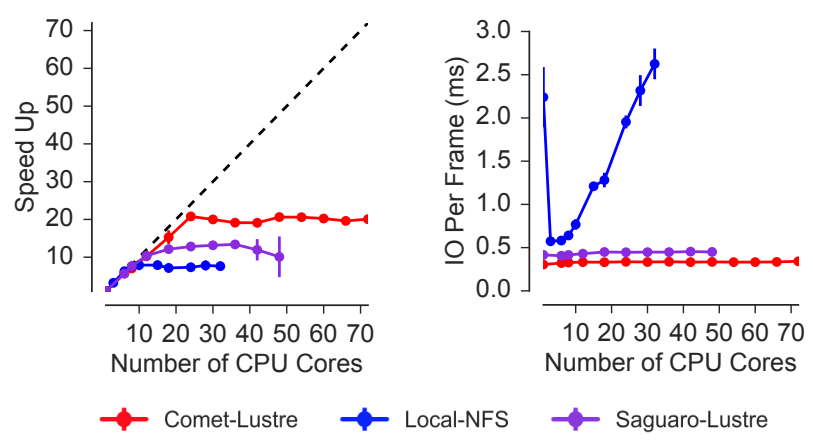

Fig. 11: Effect of three-fold over-subscribing distributed workers. The XTC600x trajectory was analyzed on HPC resources (Lustre stripe count of three) and local NFS using Dask distributed where $M$ number of trajectory blocks (tasks) is three times the number of worker processes, $M=3 N$, and there is one worker per CPU core. A Speed-up $S$. B I/O time $t_{I / O}$ per frame.
A

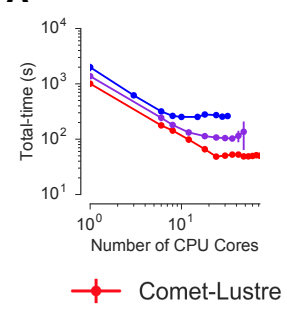

B

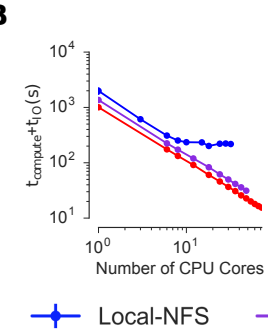

C

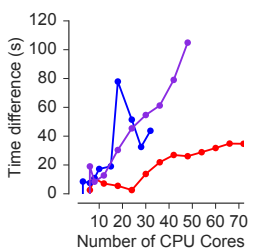

$\uparrow$ Saguaro-Lustre
Fig. 12: Detailed timings for three-fold over-subscribing distributed workers. A Total time to solution (wall clock), $t_{N}$. B $t_{c o m p}+t_{I / O}$, average sum of $t_{I / O}($ Fig. $11 \mathrm{~B})$ and the (constant) computation time $t_{\text {comp }}$ (data not shown) per frame. C Difference $t_{N}-n_{\text {frames }}\left(t_{I / O}+t_{\text {comp }}\right)$, accounting for communications and overheads that are not directly measured. Other parameters as in Fig. 11.

spent on the calculation of RMSD arrays and I/O) (computation time) which decreases as the number of cores increases from 1 to 72 . However, when extending to multiple nodes the time for overheads and communication increases, which reduces the overall performance.

In order to better quantify the scheduling decisions and to have verification of stragglers independent from the Dask web interface, we implemented a Dask scheduler reporter plugin (freely available from https:/github.com/radical-cybertools/midas), which captures task execution events from the scheduler and their respective timestamps. We analyzed the execution of XTC300x on TACC Stampede with three-fold over-subscription $\left(M=3 N_{\text {cores }}\right)$ and measured how many tasks were submitted per worker process. Table 1 shows that although most workers executed three tasks as would be expected for three-fold over-subscription, between 0 and $17 \%$ executed four tasks and others only one or two. This variability is also borne out in detail by Figure 14, which shows how RMSD blocks were submitted per worker process in each run. Therefore, over-subscription does not necessarily lead to a balanced execution and might add additional execution time; unfortunately, over-subscription does not get rid of the straggler tasks.

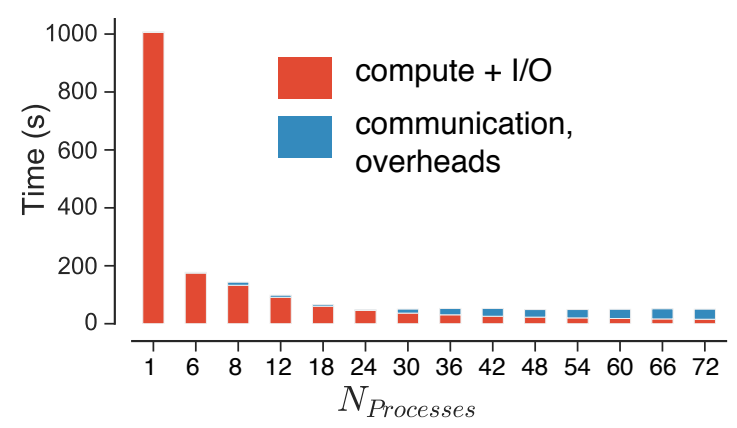

Fig. 13: Time comparison for three-fold over-subscribing distributed workers (XTC600x on SDSC Comet on Lustre with stripe count three). Bars indicate the mean total execution time $t_{N}$ (averaged over five repeats) as a function of available worker processes, with one worker per CPU core. Time for compute + I/O (red, see Fig. 12 B) dominates for smaller core counts (up to one node, 24) but is swamped by communication (time to gather the RMSD arrays computed by each worker for the reduction) and overheads (blue, see see Fig. 12 C) beyond a single node.

\begin{tabular}{llllll}
\hline $\begin{array}{l}\text { RMSD } \\
\text { Blocks }\end{array}$ & Run 1 & Run 2 & Run 3 & Run 4 & Run 5 \\
\hline 1 & 0 & 0 & 1 & 0 & 0 \\
2 & 8 & 5 & 7 & 7 & 2 \\
3 & 48 & 54 & 47 & 50 & 60 \\
4 & 8 & 5 & 9 & 7 & 2 \\
\hline
\end{tabular}

TABLE 1: Number of worker processes that executed 1, 2, 3, or 4 of tasks (RMSD calculation over one trajectory block) per run. Executed on TACC Stampede utilizing 64 cores

\section{Comparison of Performance of Map-Reduce Job Between MPI for Python and Dask Frameworks}

The investigations so far indicated that stragglers are responsible for poor scaling beyond a single node. These delayed processes were observed on three different HPC systems and on different days, so they are unlikely to be infrastructure specific. In order to rule out the hypothesis that Dask is inherently limited in its applicability to our problem we re-implemented our mapreduce problem with MPI based on the Python mpi4py [DPS05], [DPKC11] module. The comparison was performed with the XTC600x trajectory on SDSC Comet.

The overall performance is very similar to the Dask implementation: it scales almost ideally up to $24 \mathrm{CPU}$ cores (a single node)
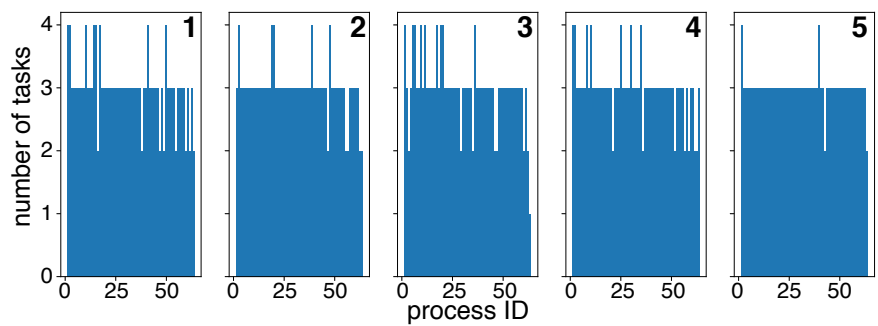

Fig. 14: Task Histogram of RMSD with MDAnalysis and Dask with XTC 300x over 64 cores on Stampede with 192 trajectory blocks. Each histogram corresponds to an independent repeat of the same computational experiment. For each worker process ID, the number of tasks submitted to that process is shown. 

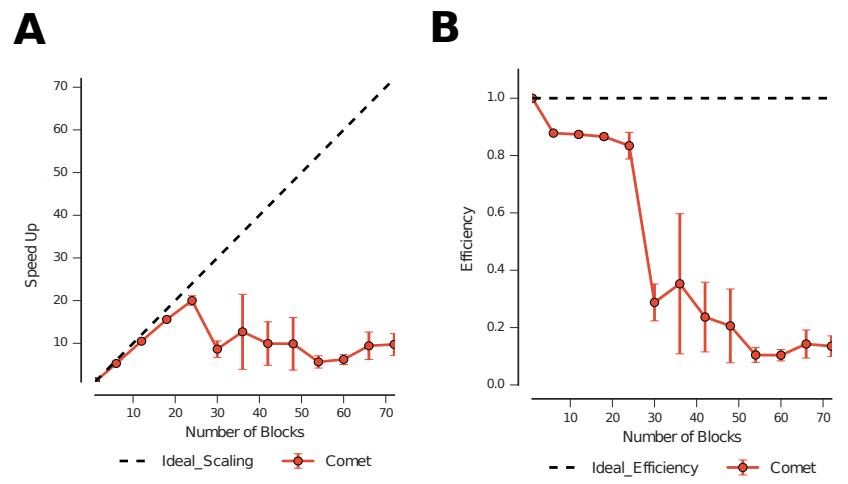

Fig. 15: A Speed-up and B efficiency plots for benchmark performed on XTC600x on SDSC Comet using MPI for Python. Five repeats are run for each block size and the reported values are the mean values and standard deviations.

but then drops to a very low efficiency (Figure 15). A detailed analysis of the time spent on computation versus communication (Figure $16 \mathrm{~A}$ ) shows that the communication and overheads are negligible up to 24 cores (single node) and only moderately increases for larger $N$. The largest fraction of the calculations is always spent on the calculation of RMSD arrays with I/O (computation time). Although the computation time decreases with increasing number of cores for a single node, it increases again when increasing $N$ further, in a pattern similar to what we saw earlier for Dask.

Figure $16 \mathrm{~B}$ compares the execution times across all MPI ranks for 72 cores. There are several processes that are about ten times slower than the majority of processes. These stragglers reduce the overall performance and are always observed when the number of cores is more than 24 and the ranks span multiple nodes. Based on the results from MPI for Python, Dask is probably not responsible for the occurrence of the stragglers.

We finally also wanted to ascertain that variable execution time is not a property of the computational task itself and replaced the RMSD calculation with optimal superposition (based on the iterative qcprot algorithm [LAT10]) with a completely different, fully deterministic metric, namely a simple all-versus-all distance calculation based on MDAnalysis.lib.distances.distance_array. The distance array calculates all distances between the reference coordinates at time 0 and the coordinates of the current frame and provides a comparable computational load. Even with the new metric the same behavior was observed in the MPI implementation (data not shown) and hence we can conclude that the qcprot RMSD calculation is not the reason why we are seeing the stragglers.

\section{Conclusions}

Dask together with MDAnalysis makes it straightforward to implement parallel analysis of MD trajectories within a mapreduce scheme. We show that obtaining good parallel performance depends on multiple factors such as storage system and trajectory file format and provide guidelines for how to optimize trajectory analysis throughput within the constraints of a heterogeneous research computing environment. Performance on a single node can be close to ideal, especially when using the XTC trajectory format that trades I/O for CPU cycles through aggressive compression, or when using SSDs with any format. However, obtaining

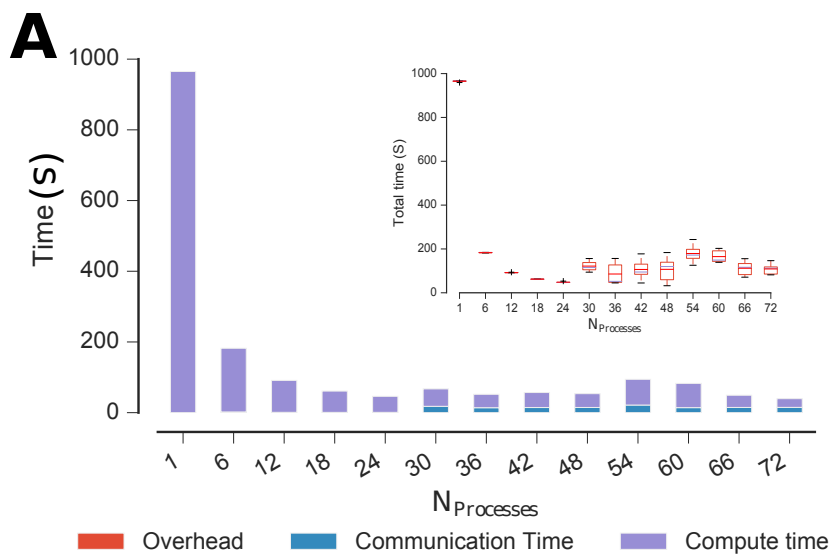

B

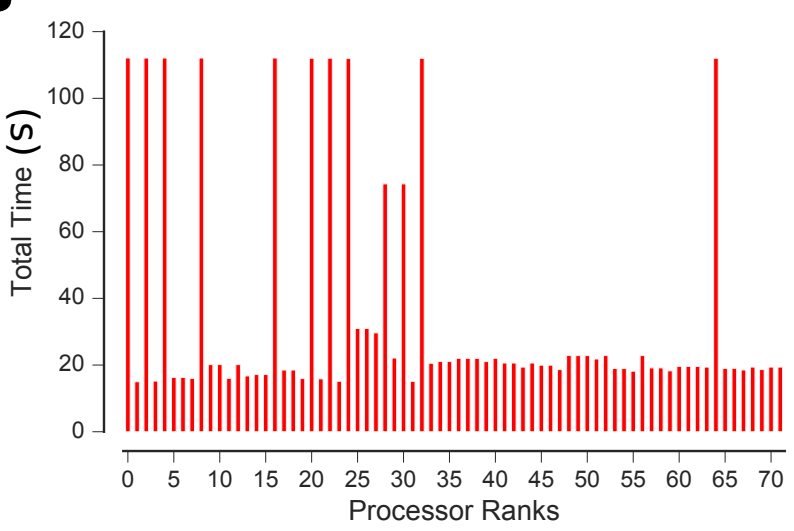

Fig. 16: A Time comparison on different parts of the calculations obtained using MPI for Python. In this aggregate view, the time spent on different parts of the calculation are combined for different number of processes tested. The bars are subdivided into different contributions (compute (RMSD computation and I/O), communication, remaining overheads), with the total reflecting the overall run time. Reported values are the mean values across 5 repeats. A inset Total job execution time along with the mean and standard deviations across 5 repeats. The calculations are performed on XTC 600x using SDSC Comet. B Comparison of job execution time across processor ranks for 72 CPU cores obtained using MPI for python. There are several stragglers that slow down the whole process.

good strong scaling beyond a single node was hindered by the occurrence of stragglers, one or few tasks that would take much longer than all the other tasks. Further studies are necessary to identify the underlying reason for the stragglers observed here; they are not due to Dask or the specific computational test case, and they cannot be circumvented by over-subscribing. Thus, implementing robust parallel trajectory analysis that scales over many nodes remains a challenge.

\section{Acknowledgments}

MK and IP were supported by grant ACI-1443054 from the National Science Foundation. SJ and OB were supported in part by grant ACI-1443054 from the National Science Foundation. Computational resources were in part provided by the Extreme Science and Engineering Discovery Environment (XSEDE), which is supported by National Science Foundation grant number ACI-1053575 (allocation MCB130177 to OB and allocation TGMCB090174 to SJ) and by Arizona State University Research Computing. 
References

[AMS ${ }^{+}$15] Mark James Abraham, Teemu Murtola, Roland Schulz, Szilárd Páll, Jeremy C. Smith, Berk Hess, and Erik Lindahl. GROMACS: High performance molecular simulations through multi-level parallelism from laptops to supercomputers. SoftwareX, 1-2:19 - 25, 2015. URL: http://www.gromacs.org, doi:10.1016/j.softx.2015.06.001.

$\left[\mathrm{BBIM}^{+}\right.$09] B R Brooks, C L Brooks III., A D Jr Mackerell, L Nilsson, R J Petrella, B Roux, Y Won, G Archontis, C Bartels, S Boresch, A Caflisch, L Caves, Q Cui, A R Dinner, M Feig, S Fischer, J Gao, M Hodoscek, W Im, K Kuczera, T Lazaridis, J Ma, V Ovchinnikov, E Paci, R W Pastor, C B Post, J Z $\mathrm{Pu}, \mathrm{M}$ Schaefer, B Tidor, R M Venable, H L Woodcock, $\mathrm{X}$ Wu, W Yang, D M York, and M Karplus. CHARMM: the biomolecular simulation program. J Comput Chem, 30(10):1545-1614, Jul 2009. URL: https://www.charmm.org, doi: $10.1002 /$ jcc.21287.

$\left[\mathrm{CCD}^{+} 05\right] \quad$ David A Case, Thomas E Cheatham, 3rd, Tom Darden, Holger Gohlke, Ray Luo, Kenneth M Merz, Jr, Alexey Onufriev, Carlos Simmerling, Bing Wang, and Robert J Woods. The amber biomolecular simulation programs. J Comput Chem, 26(16):1668-1688, 2005. URL: http://ambermd.org/, doi: $10.1002 /$ jcc. 20290.

[CR15] T. Cheatham and D. Roe. The impact of heterogeneous computing on workflows for biomolecular simulation and analysis. Computing in Science Engineering, 17(2):30-39, 2015. doi:10.1109/MCSE.2015.7.

[DG08] Jeffrey Dean and Sanjay Ghemawat. Mapreduce: simplified data processing on large clusters. Communications of the ACM, 51(1):107-113, 2008. doi:10.1145/1327452. 1327492.

[DPKC11] Lisandro D. Dalcin, Rodrigo R. Paz, Pablo A. Kler, and Alejandro Cosimo. Parallel distributed computing using python. Advances in Water Resources, 34(9):1124 - 1139, 2011. New Computational Methods and Software Tools. doi:10.1016/ j.advwatres.2011.04.013.

[DPS05] Lisandro Dalcín, Rodrigo Paz, and Mario Storti. Mpi for python. Journal of Parallel and Distributed Computing, 65(9):1108 - 1115, 2005. doi:10.1016/j.jpdc.2005. 03.010.

[FS02] Daan Frenkel and Berend Smit. Understanding Molecular Simulations. Academic Press, San Diego, 2 edition, 2002.

$\left[\mathrm{GLB}^{+} 16\right]$ Richard J. Gowers, Max Linke, Jonathan Barnoud, Tyler J. E. Reddy, Manuel N. Melo, Sean L. Seyler, David L Dotson, Jan Domański, Sébastien Buchoux, Ian M. Kenney, and Oliver Beckstein. MDAnalysis: A Python package for the rapid analysis of molecular dynamics simulations. In Sebastian Benthall and Scott Rostrup, editors, Proceedings of the 15th Python in Science Conference, pages 102 - 109, Austin, TX, 2016. SciPy. URL: http://mdanalysis.org.

[KB17] Mahzad Khoshlessan and Oliver Beckstein. Parallel analysis in the MDAnalysis library: Benchmark of trajectory file formats. Technical report, Arizona State University, Tempe, AZ, 2017. doi:10.6084/m9.figshare.4695742.

[LAT10] Pu Liu, Dimitris K Agrafiotis, and Douglas L. Theobald. Fast Determination of the Optimal Rotational Matrix for Macromolecular Superpositions. J Comput Chem, 31(7):1561-1563, 2010. doi:10.1002/jcc.21439.

[MADWB11] Naveen Michaud-Agrawal, Elizabeth Jane Denning, Thomas B. Woolf, and Oliver Beckstein. MDAnalysis: A toolkit for the analysis of molecular dynamics simulations. J Comput Chem, 32:2319-2327, 2011. URL: http://mdanalysis.org, doi:10 . $1002 /$ jcc. 21787.

[MM14] Cameron Mura and Charles E. McAnany. An introduction to biomolecular simulations and docking. Molecular Simulation, 40(10-11):732-764, 2014. doi:10.1080/ 08927022.2014 .935372 .

[RCI13] Daniel R. Roe and Thomas E. Cheatham III. PTRAJ and CPPTRAJ: Software for processing and analysis of molecular dynamics trajectory data. J Chemical Theory Computation, 9(7):3084-3095, 2013. URL: https://github.com/Amber-MD/ cpptraj, doi:10.1021/ct 400341 p.

[Roc15] Matthew Rocklin. Dask: Parallel computation with blocked algorithms and task scheduling. In Proceedings of the 14th Python in Science Conference, number 130-136, 2015. URL: https://github.com/dask/dask.
[SB14]

Sean L Seyler and Oliver Beckstein. Sampling of large conformational transitions: Adenylate kinase as a testing ground. Molec. Simul., 40(10-11):855-877, 2014. doi:10.1080/ 08927022.2014 .919497$.

[SB17] Sean Seyler and Oliver Beckstein. Molecular dynamics trajectory for benchmarking MDAnalysis, 62017 URL: https://figshare.com/articles/Molecular_dynamics_ trajectory_for_benchmarking_MDAnalysis/5108170, doi:10.6084/m9.figshare. 5108170

$\left[\mathrm{TRB}^{+} 08\right]$ T. Tu, C.A. Rendleman, D.W. Borhani, R.O. Dror, J. Gullingsrud, MO Jensen, J. L. Klepeis, P. Maragakis, P. Miller, K.A. Stafford, and David E. Shaw. A scalable parallel framework for analyzing terascale molecular dynamics simulation trajectories. In International Conference for High Performance Computing, Networking, Storage and Analysis, 2008. SC 2008., pages 1-12, Austin, TX, 2008. IEEE. doi:10.1109/SC.2008.5214715.

[VCV11] Stefan Van Der Walt, S. Chris Colbert, and Gael Varoquaux. The NumPy array: A structure for efficient numerical computation. Comput Sci Eng, 13(2):22-30, 2011. URL: http: //www.numpy.org/, arXiv:1102.1523, doi:10.1109/ MCSE.2011.37. 